\title{
First results of baryon interactions from lattice QCD with physical masses (3) - Strangeness $S=-2$ two-baryon system
}

\author{
Kenji Sasaki ${ }^{* a}$, Sinya Aoki ${ }^{b}$, Takumi Doi ${ }^{c}$, Shinya Gongyo ${ }^{b}$, Tetsuo Hatsuda ${ }^{c d}$, \\ Yoichi Ikeda $^{c}$, Takashi Inoue $^{e}$, Takumi Iritani $^{f}$, Noriyoshi Ishii $^{g}$, Takaya Miyamoto $^{b}$, \\ Keiko Murano ${ }^{g}$ \\ ${ }^{a}$ Center for Computational Sciences, University of Tsukuba, Ibaraki 305-8571, Japan \\ ${ }^{b}$ Yukawa Institute for Theoretical Physics, Kyoto University, Kyoto 606-8502, Japan \\ ${ }^{c}$ Theoretical Research Division, Nishina Center, RIKEN, Wako 351-0198, Japan \\ ${ }^{d}$ Kavli IPMU (WPI), The University of Tokyo, Chiba 277-8583, Japan \\ ${ }^{e}$ Nihon University, College of Bioresource Sciences, Kanagawa 252-0880, Japan \\ ${ }^{f}$ Department of Physics and Astronomy, Stony Brook University, Stony Brook, New York \\ 11794-3800, USA \\ ${ }^{g}$ Research Center for Nuclear Physics (RCNP), Osaka University, Osaka 567-0047, Japan \\ E-mail: kenjis@het.ph.tsukuba.ac.jp
}

\begin{abstract}
We investigate the strangeness $S=-2$ two-baryon system by lattice QCD theory at nearly physical point with a huge lattice volume of $L a=8.2 \mathrm{fm}$ generated on the K computer at AICS. Our approach (HAL QCD method) enable us to derive a potential from coupled channel Schrödinger equation using the Nambu-Bethe-Salpeter (NBS) wave functions. We report our latest results of the $S=-2$ baryon-baryon interactions focusing on the ${ }^{1} S_{0}$ with $I=0$ channel where the Hdibaryon state is predicted by several model calculations.
\end{abstract}

The 33rd International Symposium on Lattice Field Theory

14 -18 July 2015

Kobe International Conference Center, Kobe, Japan*

* Speaker. 


\section{Introduction}

Low-energy scattering observables of two baryons are essential to determine the baryonbaryon interactions which are important inputs for (hyper-)nuclear physics. Theoretical studies of the strangeness $S=-2$ two-baryon system where possibility of the $H$-dibaryon is discussed $[1,2]$ are significant because experimental data are quite limited. However it has been thought to be impossibly difficult to solve the dynamics of quarks and gluons from QCD because of its non-perturbative nature at low-energies.

Lattice QCD approach enables us to tackle the problem non-perturbatively by numerical simulations. In the framework, M. Lüscher firstly proposed to relate the energy $E$ of a two-particle state in a finite box to the elastic scattering phase $\delta(E)$ in the continuum [3]. The relation is derived by using the asymptotic behavior of the two-particle Nambu-Bethe-Salpeter (NBS) wave function $\psi(r)$, if the range of the interaction is sufficiently smaller than the size of the box.

An alternative approach to the hadron interactions from lattice QCD has been proposed $[4,5]$ and has been extensively developed by the HAL QCD Collaboration $[6,7,8,9,10,11,12,13,14]$, called the HAL QCD method. In the method, we define the energy-independent and non-local potential $U\left(r, r^{\prime}\right)$ from $\psi(r)$ which obeys the Schrödinger type equation in a finite box. Using the obtained $U$ which receives only weak finite volume effect, we can simply calculate the scattering phase shifts and bound state spectra in infinite space to compare the results with experimental data.

A further advantage of the HAL QCD method is that it can be generalized straightforwardly to the case of inelastic scatterings. As discussed in the next section, we straightforwardly construct the coupled channel Schrödinger equation once all corresponding NBS wave functions are obtained. Once such a potential is constructed in the finite volume, we can calculate any scattering observebles which one needs by solving the Schrödinger equation in the infinite volume.

The $B B$ interactions in the flavor $\mathrm{SU}(3)$ limit have been studied systematically in full QCD simulations on the lattice by the HAL QCD method for several masses of the pseudo-scalar meson $m_{\mathrm{PS}}=470-1170 \mathrm{MeV}$ [9]. In such situation, the H-dibayon was found in flavor singlet state for all quark masses. In this paper, we investigate the $B B$ interaction in the $H$-dibaryon channel with the explicit SU(3) breaking on the basis of the coupled channel HAL QCD method developed in our previous works[7, 14].

\section{Coupled channel $B B$ potentials}

In this section, we briefly define coupled channel potentials in the HAL QCD method following Ref. [7] considering the $S=-2 B B$ system as $\Lambda \Lambda \leftrightarrow N \Xi \leftrightarrow \Sigma \Sigma$ inelastic scatterings.

We first introduce the normalized 4-pt correlation function $R$ in channel $c$ defined as

$$
R_{\mathscr{I}_{d}}^{c}(\vec{r}, t) \equiv \frac{\left\langle 0\left|B_{c_{1}}(\vec{x}+\vec{r}, t) B_{c_{2}}(\vec{x}, t) \overline{\mathscr{I}}_{d}\left(t_{0}=0\right)\right| 0\right\rangle}{\sqrt{Z_{c_{1}} Z_{c_{2}}} \exp \left[-\left(m_{c_{1}}+m_{c_{2}}\right) t\right]}=\sum_{n} \psi_{W_{n}}^{c}(\vec{r}) e^{-\Delta W_{n}^{c} t} A_{d}^{W_{n}}+\cdots,
$$

where $\Delta W_{n}^{c}=W_{n}-m_{c_{1}}-m_{c_{2}}$ with the total energy $W_{n}$ and baryon mass $m_{c_{i}}$, and $A_{d}^{W_{n}}=\left\langle W_{n}\left|\bar{I}_{d}(0)\right| 0\right\rangle$. The ellipses in Eq.(2.1) denote inelastic contributions from channels which we are not considering. The equal-time NBS wave function $\psi_{W_{n}}^{c}$ is embedded in the $R$-function. An interpolating operator $B_{c_{j}}(\vec{x}, t)$ with a channel index, $c$, and particle index, $j=1,2$, denotes a local composite operator for 
octet baryons with its wave-function renormalization factor $\sqrt{Z_{c_{j}}}$. The corresponding asymptotic momentum $k_{i}^{c}$ in the center-of-mass $(\mathrm{CM})$ frame is defined through the relation,

$$
W_{n}=\sqrt{m_{c_{1}}^{2}+\left(k_{n}^{c}\right)^{2}}+\sqrt{m_{c_{2}}^{2}+\left(k_{n}^{c}\right)^{2}} .
$$

In the non-relativistic approximation valid at low energies, we can replace the kinetic energy term in the equation with the time derivative as

$$
-\frac{\partial}{\partial t} R^{c} \mathscr{I}_{d}(\vec{r}, t)=\sum_{n} \Delta W_{n}^{c} \psi_{W_{n}}^{c}(\vec{r}) e^{-\Delta W_{n} t} A_{d}^{W_{n}}
$$

with which we obtain the Schrödinger type equation,

$$
\left(-\frac{\partial}{\partial t}-H_{0}^{c}\right) R^{c} \mathscr{I}_{d}(\vec{r}, t)=\int d^{3} r^{\prime} U_{e}^{c}\left(\vec{r}, \vec{r}^{\prime}\right) \Delta^{c} R^{e} \mathscr{\mathscr { I }}_{d}\left(\vec{r}^{\prime}, t\right),
$$

where $H_{0}^{c}=-\frac{\nabla^{2}}{2 \mu^{c}}$ and $\Delta^{c} e=\exp \left[-\left(m_{e_{1}}+m_{e_{2}}\right) t\right] / \exp \left[-\left(m_{c_{1}}+m_{c_{2}}\right) t\right]$. If we go beyond the nonrelativistic approximation, higher-order time-derivatives appear, which we will not consider in this paper. In order to control the non-locality of the potential, the derivative expansion is carried out as $U\left(\vec{r}, \vec{r}^{\prime}\right)=\left(V_{\mathrm{LO}}(\vec{r})+V_{\mathrm{NLO}}(\vec{r})+\cdots\right) \delta\left(\vec{r}-\vec{r}^{\prime}\right)$, where $\mathrm{N}^{n} \mathrm{LO}$ term is of $O\left(\vec{\nabla}^{n}\right)$ and its convergence has been confirmed for the $N N$ case [8].

The flavor structures of baryons are given as

$$
\begin{aligned}
& S=0 \quad I=1 / 2: p=[u d] u, \quad n=[u d] d \\
& S=-1 \quad I=1 \quad: \Sigma^{+}=-[u s] u, \Sigma^{-}=-[d s] d, \Sigma^{0}=-\frac{1}{\sqrt{2}}([d s] u+[u s] d) \\
& S=-1 I=0 \quad: \Lambda=\frac{1}{\sqrt{6}}([s d] u+[u s] d-2[d u] s) \\
& S=-2 I=1 / 2: \Xi^{0}=[s u] s, \quad \Xi^{-}=[s d] s
\end{aligned}
$$

where a square bracket stands for an antisymmetric combination. Focusing on the $H$-dibaryon channel in the $S=-2$ system, we only consider the $I=0$ channel due to the Fermi-Dirac statistics of two baryons. They are defined as

$$
(\Lambda \Lambda)_{I=0}=\Lambda \Lambda,(N \Xi)_{I=0}=\sqrt{\frac{1}{2}}\left(p \Xi^{-}-n \Xi^{0}\right),(\Sigma \Sigma)_{I=0}=\sqrt{\frac{1}{3}}\left(\Sigma^{+} \Sigma^{-}+\Sigma^{-} \Sigma^{+}-\Sigma^{0} \Sigma^{0}\right) .
$$

The relation between particle basis and $\mathrm{SU}(3)$ basis is given by $\mathrm{SU}(3)$ coefficients as

$$
\left|\begin{array}{l}
\mathbf{8} \\
\mathbf{2}
\end{array}\right\rangle=\left(\begin{array}{ccc}
-\sqrt{\frac{1}{8}} & \sqrt{\frac{1}{2}} & \sqrt{\frac{3}{8}} \\
-\sqrt{\frac{1}{5}} & \sqrt{\frac{1}{5}} & -\sqrt{\frac{3}{5}} \\
\sqrt{\frac{27}{40}} & \sqrt{\frac{3}{10}} & -\sqrt{\frac{1}{40}}
\end{array}\right)\left|\begin{array}{l}
\Lambda \Lambda \\
N \Xi \\
\Sigma \Sigma
\end{array}\right\rangle
$$

for ${ }^{1} S_{0}(I=0)$ (H-dibaryon) channel.

\section{Lattice setup}

We employ $N_{f}=2+1$ gauge configurations are generated with the Iwasaki gauge action at $\beta=1.82$ and nonperturbatively $\mathscr{O}(a)$-improved Wilson quark action with $c_{s w}=1.11$ on the 
Table 1: Baryon masses in units of $\mathrm{MeV}$.

\begin{tabular}{c|cccc}
\hline \hline particle & $N$ & $\Lambda$ & $\Sigma$ & $\Xi$ \\
\hline mass $[\mathrm{MeV}]$ & $956 \pm 11$ & $1124 \pm 3$ & $1206 \pm 4$ & $1330 \pm 1$ \\
\hline \hline
\end{tabular}

$L^{3} \times T=96^{3} \times 96$ lattice. The hopping parameters for light (ud) and strange quarks are choosen as $\left(\kappa_{u d}, \kappa_{s}\right)=(0.126117,0.124790)$ corresponding to $m_{\pi} \simeq 146 \mathrm{MeV}$ and $m_{K} \simeq 525 \mathrm{MeV}$ with $a^{-1} \simeq$ $2.33 \mathrm{GeV}(a \simeq 0.085 \mathrm{fm})$. The total statistics used in this report amounts to 200 configurations $\times 4$ rotations $\times 20$ wall sources. This lattice setup brings about the almost physical point simulation of the $B B$ interaction on the large lattice volume of $(8.2 \mathrm{fm})^{4}$ where a finite volume effects of the $B B$ potential could be neglected. The calculated baryon masses in this setup are listed in Tab. 3.

Quark propagators are calculated in consideration of the spatial wall source by imposing Coulomb gauge fixing at $t_{0}=0$ with the Dirichlet boundary condition in temporal direction at $t=48$. The forward and backward propagations of baryon 4-pt correlator are averaged and four rotated gauge configurations are used to reduce the statistical errors. An average over the cubic group is taken for the sink operator to project on the S-wave in the $B B$ wave function.

\section{Results and discussions}

We now present our results of coupled channel $B B$ potentials in strangeness $S=-2$ sector. The potential matrix elements $V^{i}{ }_{j} \mathrm{~s}$ are calculated by using the NBS wave functions at $t=10^{1}$.
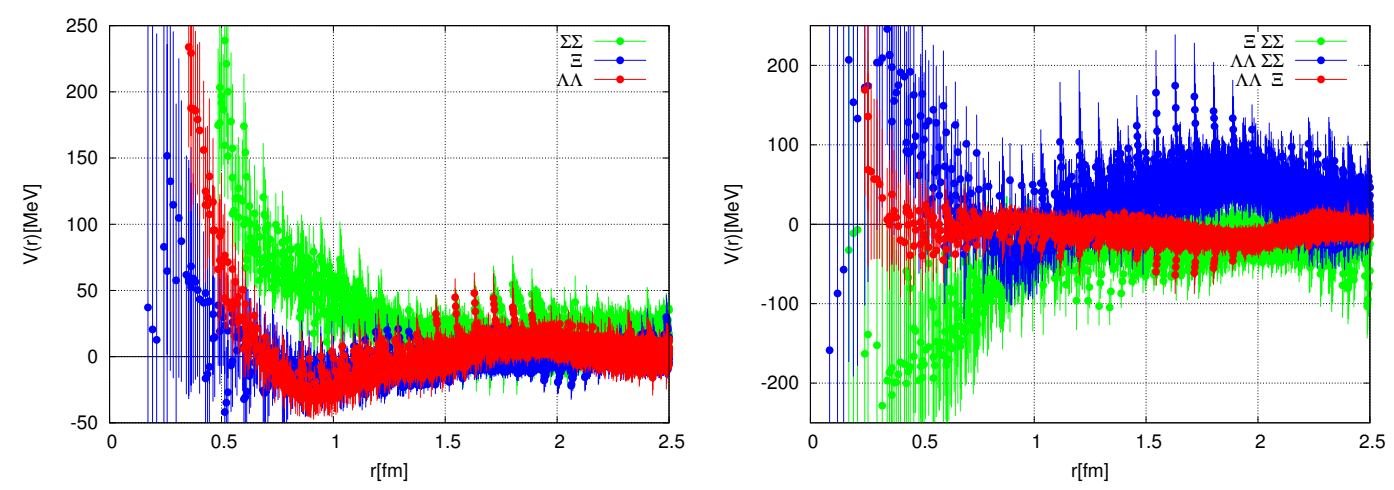

Figure 1: The $\Lambda \Lambda$ (red), $N \Xi$ (blue) and $\Sigma \Sigma$ (green) potentials are shown in the left panel. The $\Lambda \Lambda-N \Xi$ (red), $\Lambda \Lambda-N \Xi$ (blue) and $N \Xi-\Sigma \Sigma$ (green) transition potentials are shown in the right panel.

Potentials in ${ }^{1} S_{0}$ with $S=-2$ and $I=0$, in which $\Lambda \Lambda, N \Xi$ and $\Sigma \Sigma$ channels are coupled, are shown in Fig. 1. In the left panel, we find that all diagonal elements of the potential matrix have a repulsive core at short distances, whose strength, however, strongly depends on its state. On the other hand, an attractive pocket appears only in two diagonal elements, $V^{\Lambda \Lambda}{ }_{\Lambda \Lambda}$ and $V^{N \Xi}{ }_{N \Xi}$, where $V^{N \Xi}{ }_{N \Xi}$ has relatively weaker repulsive core than $V^{\Lambda \Lambda}{ }_{\Lambda \Lambda}$ does, while $V^{\Sigma \Sigma}{ }_{\Sigma \Sigma}$ is totally repulsive in

\footnotetext{
${ }^{1}$ We will increase $t$ to suppress inelastic contributions, as statistical errors will be decreased in future.
} 
the whole range of $r$. Among the off-diagonal elements given in the right panel in Fig. 1, $V^{\Lambda \Lambda} \equiv$ is smaller than other two, so that the decay rate from $N \Xi$ to $\Lambda \Lambda$ may be rather suppressed.
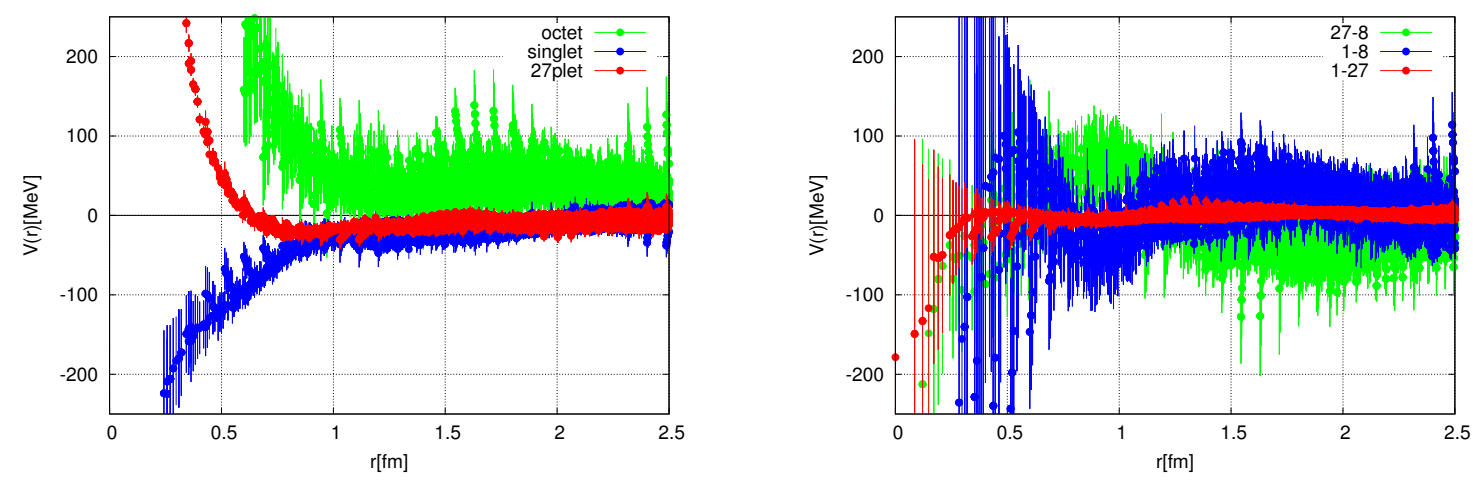

Figure 2: Potential matrix in the $\mathrm{SU}(3)$ basis for ${ }^{1} S_{0}$ with $S=-2(I=0)$. The diagonal elements, $V^{1}{ }_{1}$ (blue), $V^{8}{ }_{8}$ (green) and $V^{27}{ }_{27}(\mathrm{red})$ are shown in the left panel, while panel off-diagonal ones, $V^{1}{ }_{8}$ (blue), $V^{1}{ }_{27}$ (red) and $V^{8}{ }_{27}$ (green) in the right panel.

It is instructive to observe the potentials in the basis of the SU(3) irreducible representations which is obtained by using Clebsch-Gordan coefficients given in eq. (2.7). The potential matrix in the SU(3) basis for ${ }^{1} S_{0}(I=0)$ is given in Fig. 2. As in the case of the SU(3) limit [9], the diagonal element for the flavor singlet state, $V_{1}^{1}$, is strongly attractive, while $V_{8}^{8}$ is repulsive at all distances with relatively large statistical errors. The attractive nature of the flavor singlet basis is consistent with the absence of the quark Pauli blocking effect and the attractive color-magnetic interaction for quarks predicted in Ref. [15]. For the potential in 27-plet basis, we find a familiar shape of potential to nuclear potential, a short-range repulsive core is surrounded by an attractive pocket. It is easily understood because the $N N\left({ }^{1} S_{0}\right)$ channel belongs to the same multiplet in the $\mathrm{SU}(3)$ symmetric limit.

Transition potentials between different SU(3) bases are effective measure for the symmetry breaking effects because such a transition never happens if we consider the exact flavor symmetry. The right panel in Fig. 2 shows that off-diagonal potentials are consistent to be zero within errorbars in whole region, so that the symmetry breaking effects might not be so large even in the nearly physical point simulation.
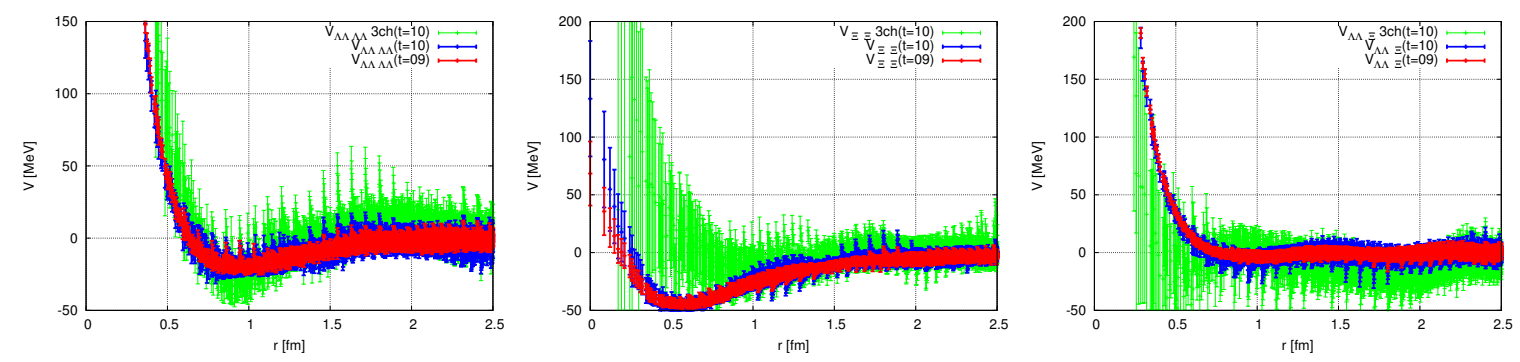

Figure 3: Effective $\Lambda \Lambda$ and $N \Xi$ coupled channel potential. The original potentials by three-channel calculation are also shown for comparison.

Up to this point, we look over the potential matrix in the H-dibaryon channel which is not suitable to calculate any scattering observables due to large statistical noises. To reduce the errors, 
we attempt to evaluate an effective coupled channel potential by integrating out the $\Sigma \Sigma$ state. The effective potential is valid below the $\Sigma \Sigma$ threshold up to non-locality error. Under this situation, the effective potential matrix $\tilde{V}_{j}^{i}$ is obtained as

$$
\begin{aligned}
& \left(\begin{array}{ll}
\tilde{V}_{\Lambda \Lambda}^{\Lambda \Lambda}(\vec{r}) & \tilde{V}_{N \Xi}^{\Lambda \Lambda}(\vec{r}) \Delta^{\Lambda \Lambda}{ }_{N \Xi} \\
\tilde{V}_{\Lambda \Lambda}^{N \Xi}(\vec{r}) \Delta^{N \Xi} & \tilde{V}_{N \Xi}^{N \Xi}(\vec{r})
\end{array}\right) \\
& \quad \simeq\left(\begin{array}{cc}
\left(-\frac{\partial}{\partial t}-H_{0}^{\Lambda \Lambda}\right) R_{\mathscr{I}_{\Lambda \Lambda}}^{\Lambda \Lambda}(\vec{r}, t) & \left(-\frac{\partial}{\partial t}-H_{0}^{\Lambda \Lambda}\right) R_{\mathscr{I}_{N \Xi}}^{\Lambda \Lambda}(\vec{r}, t) \\
\left(-\frac{\partial}{\partial t}-H_{0}^{N \Xi}\right) R_{\mathscr{I}_{\Lambda \Lambda}}^{N \Xi}(\vec{r}, t) & \left(-\frac{\partial}{\partial t}-H_{0}^{N \Xi}\right) R_{\mathscr{I}_{N \Xi}}^{N \Xi}(\vec{r}, t)
\end{array}\right)\left(\begin{array}{c}
R_{\mathscr{I}_{\Lambda \Lambda}}^{\Lambda \Lambda}(\vec{r}, t) R_{\mathscr{I}_{N \Xi}(\vec{r}, t)}^{\Lambda R_{\mathscr{I}_{\Lambda \Lambda}}^{N \Xi}(\vec{r}, t)} R_{\mathscr{I}_{N \Xi} \Xi}^{N \Xi}(\vec{r}, t)
\end{array}\right)^{-1} .
\end{aligned}
$$

Fig. 3 shows the effective $\Lambda \Lambda$ and $N \Xi$ coupled channel potential. Although the effective $\Lambda \Lambda$ potential is similar to the original one, the effective $N \Xi$ potential becomes deeper comparing to the original $N \Xi$ potential and its errorbars are largely reduced. Transition potential, whose errorbars are also reduced considerably, is relatively stronger than the original one at short distances. The main differences can be only seen in $r<0.7 \mathrm{fm}$ which means that the channel truncation effects would emerge in high momentum scattering regions.
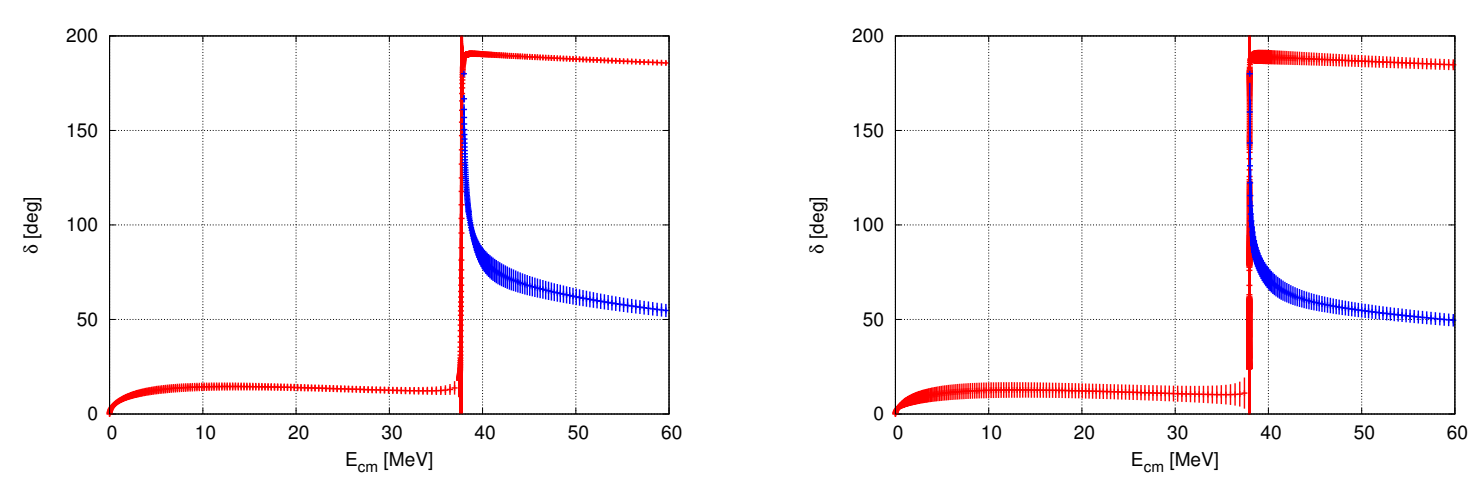

Figure 4: Preliminary results of $\Lambda \Lambda$ and $N \Xi$ scattering phase shifts. The result with the potential at $t=9$ is given in the left panel, while one at $t=10$ is in the right panel

Fig. 4 shows the $\Lambda \Lambda$ and $N \Xi$ phase shifts by using the channel truncated potentials. We find that there is qualitatively equivalent resutlts between the phase shift at $t=9$ and $t=10$. The results show that there is a clear resonance just below the $N \Xi$ threshold. Since inclusion of $\Sigma \Sigma$ channel effects could not affect very much for low energy $\Lambda \Lambda$ phase shifts, these results implicate that a deeply bound $\mathrm{H}$-dibayon state would not be generated even in more sophisticated calculations.

\section{Conclusions}

We have investigated $S=-2$ BB interactions from lattice QCD employing $N_{f}=2+1$ gauge configurations with $(96 a)^{4}$ and $a \simeq 0.085 \mathrm{fm}$ lattice, where $m_{\pi} \simeq 146 \mathrm{MeV}$ and $m_{K} \simeq 525 \mathrm{MeV}$. Baryon potentials have been calculated by the coupled channel HAL QCD method with considerations of not only spacial but also temporal correlations of baryon 4-pt correlation functions which involves the equal-time NBS wave functions.

We find that both the $\Lambda \Lambda$ and $N \Xi$ potentials in the ${ }^{1} S_{0}$ channel have a long-range attractive pocket and a short-range repulsive core. The strength of repulsive core of $N \Xi$ potential is rather 
weaker than that of $\Lambda \Lambda$ one. We also find that the potential in flavor singlet ${ }^{1} S_{0}$ channel, which is obtained with a help of SU(3) CG coefficients, is strongly attractive which is a similar consequence of the consistent quark model. Since the obtained potentials are largely fluctuated to calculate several observables, we perform a provisional calculation of coupled $\Lambda \Lambda$ and $N \Xi$ effective potentials. The effective potentials which could be valid in the region where the energy is small enough from the $\Sigma \Sigma$ threshold yield the clear resonance behavior of the $\Lambda \Lambda$ phase shift just below the $N \Xi$ threshold.

The results in this paper are still very preliminary but further investigations will be performed with high statistics data.

\section{Acknowledgments}

The lattice QCD calculations have been performed on the K computer at RIKEN, AICS (Nos. hp120281, hp130023, hp140209, hp150223), HOKUSAI FX100 computer at RIKEN, Wako (No. G15023) and HA-PACS at University of Tsukuba (Nos. 14a-20, 15a-30). We thank ILDG/JLDG [16] which serves as an essential infrastructure in this study. This work is supported in part by MEXT Grant-in-Aid for Scientific Research (15K17667, 25287046, 26400281), and SPIRE (Strategic Program for Innovative REsearch) Field 5 project.

\section{References}

[1] R. L. Jaffe, Phys. Rev. Lett. 38 (1977) 195 [Erratum-ibid. 38 (1977) 617].

[2] T. Sakai, K. Shimizu and K. Yazaki, Prog. Theor. Phys. Suppl. 137 (2000) 121 [nucl-th/9912063].

[3] M. Lüscher, Nucl. Phys. B 354 (1991) 531.

[4] N. Ishii, S. Aoki and T. Hatsuda, Phys. Rev. Lett. 99 (2007) 022001 [nucl-th/0611096].

[5] S. Aoki et al. [HAL QCD Collaboration], Prog. Theor. Exp. Phys. (2012) 01A105 [arXiv:1206.5088 [hep-lat]].

[6] H. Nemura, N. Ishii, S. Aoki and T. Hatsuda, Phys. Lett. B 673 (2009) 136 [arXiv:0806.1094 [nucl-th]].

[7] S. Aoki et al. [HAL QCD Collaboration], Proc. Jpn. Acad., Ser. B, 87 (2011) 509 [arXiv:1106.2281 [hep-lat]].

[8] K. Murano, N. Ishii, S. Aoki and T. Hatsuda, Prog. Theor. Phys. 125 (2011) 1225. [arXiv:1103.0619 [hep-lat]].

[9] T. Inoue et al. [HAL QCD Collaboration], Nucl. Phys. A 881 (2012) 28 [arXiv:1112.5926 [hep-lat]].

[10] T. Doi et al. [HAL QCD Collaboration], Prog. Theor. Phys. 127 (2012) 723 [arXiv:1106.2276 [hep-lat]].

[11] N. Ishii et al. [HAL QCD Collaboration], Phys. Lett. B 712 (2012) 437 [arXiv:1203.3642 [hep-lat]].

[12] K. Murano et al. [HAL QCD Collaboration], Phys. Lett. B 735 (2014) 19 [arXiv:1305.2293 [hep-lat]].

[13] Y. Ikeda et al., Phys. Lett. B 729 (2014) 85 [arXiv:1311.6214 [hep-lat]].

[14] K. Sasaki et al. Prog. Theor. Exp. Phys. (2015) 113B01 [arXiv:1504.01717 [hep-lat]]. 
[15] M. Oka, K. Shimizu and K. Yazaki, Prog. Theor. Phys. Suppl. 137 (2000) 1.

[16] See "http://www.lqcd.org/ildg" and "http://www.jldg.org" 